\title{
THERMAL CHANGES IN PERIPHERAL VASCULAR DISEASE DURING SYMPATHETIC GANGLIONECTOMY UNDER GENERAL ANESTHESIA
}

\author{
BY WINCHELL MCK. CRAIG, BAYARD T. HORTON \\ AND CHARLES SHEARD \\ (From the Section on Neurologic Surgery and the Division of Medicine, The Mayo Clinic \\ and the Division of Physics and Biophysical Research, \\ The Mayo Foundation, Rochester, Minn.)
}

(Received for publication February 2, 1933)

A great deal of confusion has existed regarding the differential diagnosis of Raynaud's disease, thrombo-angiitis obliterans, and arteriosclerosis. Raynaud's disease affects, predominantly, young women, and because it is caused by vasospasm is completely relieved in the uncomplicated case, by sympathetic ganglionectomy. It need not be confused with thromboangiitis obliterans and arteriosclerosis with occlusion. Arteriosclerosis with occlusion is usually found among elderly people, and since vasospasm is rarely, if ever, an element in the condition, it is not affected by sympathetic ganglionectomy. Thrombo-angiitis obliterans practically always affects young men, has an element of vasospasm, and many of the patients can be satisfactorily treated by sympathetic ganglionectomy. Simple tests have been sought which would aid in differential diagnosis and which, by revealing the degree of vasodilation possible, would help to determine the suitability of these cases for operation, and, in some degree, indicate the probable prognosis. Observations made on patients undergoing sympathetic ganglionectomy under general anesthesia revealed certain thermal changes which may prove of value in this connection.

With the use of general anesthesia, administered by inhalation, it was noted that extremities which were not denervated were temporarily relieved of vasomotor spasm, and that there was definite postoperative clinical improvement of these extremities. It was difficult to interpret this temporary improvement, but it was thought for a time that the entire sympathetic nervous system was reflexly or indirectly affected by operation on, or manipulation of, any part. Adson (1) ventured the suggestion that the general anesthetic probably influenced the entire sympathetic nervous system by affecting the vasoconstrictor center in the diencephalon. Morton and Scott (6) measured the changes in cutaneous

${ }^{1}$ Read before the Central Society for Clinical Research, Chicago, Illinois, November 4, 1932. 
temperature caused by different anesthetic agents and called attention to the different temperature gradients in the extremities of normal people. They suggested the use of general anesthesia as a means of selecting cases suitable for sympathetic ganglionectomy, but concluded that it was not satisfactory. Sheard, Rynearson and Craig (9) have shown experimentally that in dogs under anesthesia by inhalation, generalized vasodilation occurred and that this was not influenced by operation on the sympathetic system. Also they showed that under general anesthesia the changes in the temperatures of intact and of sympathectomized extremities, with variations in environmental temperature, were similar.

Herrick, Essex and Baldes (5), using a modification of the thermostromuhr method of Rein, have shown that following unilateral sympathetic ganglionectomy on dogs, the flow in the femoral arteries on the sympathectomized side was about twice as great as that on the intact side. There was no outstanding difference between the readings of the two sides when, following the operations, these animals were placed under ether anesthesia. Hence, it may be concluded that during surgical anesthesia by ether, there develops vasodilation of the vessels of the extremities as great as that following sympathetic ganglionectomy.

Thus, it would seem that during operations on the sympathetic nervous system under anesthesia by inhalation, there occurred certain physiologic changes which could be traced directly to the anesthetic, and which were not altered by removal of ganglia. By observing the changes in cutaneous temperature during operations under general anesthesia, certain important facts were obtained which we believe will prove of diagnostic and prognostic value in treating cases of peripheral vascular disease.

This study consisted of continuous observations of surface temperature of the extremities of subjects with Raynaud's disease and thrombo-angiitis obliterans during the course of thoracic and lumbar sympathetic ganglionectomy. Repeated readings of temperature were made preoperatively with the subjects at rest and with the room temperature at approximately $24^{\circ} \mathrm{C}$. A portable electromotive thermometer, which is a modification of the ensemble described by Sheard (8), was used throughout the investigations. The instrument used carried four thermocouples for application to the hands and feet. By means of a rotary switch, any thermocouple could be placed in the circuit and the temperature indicated by each of the thermocouples could be read quickly and accurately on the micro-ammeter calibrated in degrees Centigrade. The thermocouples were left in place during the entire course of the operation, so that graphic records were obtained of the changes in surface temperature of the extremities as influenced by anesthesia alone and by the interruption of the sympathetic innervation to the extremities. The operations were carried out under general anesthesia with nitrous oxide, oxygen and ether, or ethylene, oxygen and ether. Regardless of induction and maintenance 
with gas, oxygen, or ether administered by the drop method, the relative changes in the temperature curve were the same. Observations of the blood pressure and pulse were made during this period. For a control study, similar observations of changes of surface temperature were made on subjects during the course of operation for other conditions.

The rise in surface temperature of the extremities of an apparently normal subject is illustrated in Figure 1 and represents a normal response

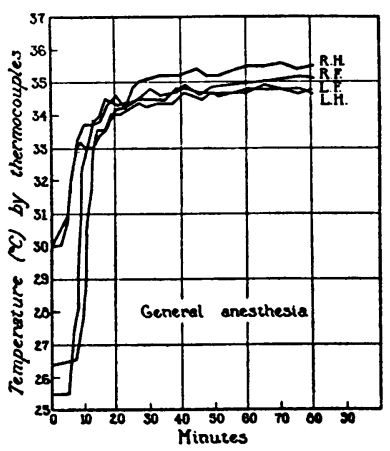

Fig. 1. Course of Thermal Changes in Hands and Feet of a Patient with Apparently Normal Vasomotor Reaction to General Anesthesia

R. H., right hand; R. F., right foot; L. F., left foot, and L. H., left hand.

of vasodilation. There was a relatively sharp and uniform rise in the surface temperature of all four extremities, within the first ten minutes after the induction of anesthesia. The maximal rise of surface temperature occurred within twenty to thirty minutes and remained constant for one hour, the pulse rate varied from 70 to 80 beats each minute, and the systolic blood pressure varied from 122 to $130 \mathrm{~mm}$. $\mathrm{Hg}$ and the diastolic, from 60 to $74 \mathrm{~mm}$. $\mathrm{Hg}$.

The marked similarity between the rise in surface temperature of the extremities in a subject with Raynaud's disease and that of the normal person is illustrated by Figure 2a. The same sharp rise in surface temperature occurred in all four extremities immediately after induction of anesthesia, and reached its maximum within twenty to twenty-five minutes. Practically no change was observed in the surface temperature of the extremities after this period. This is what one would expect in a case of Raynaud's disease, which is a primary vasospastic disturbance involving the vessels of the extremities. It is interesting to note that no change in the surface temperature of the lower extremities was observed following interruption of the sympathetic nerve supply. In other words, the maximal rise in surface temperature had occurred immediately following induction of the anesthesia, and severance of the sympathetic nerve supply caused no additional rise in surface temperature. This is of particular interest because it was formerly a clinical impression among certain 
workers that immediately following interruption of the sympathetic nerve supply to a given extremity, there was always a sharp rise of surface temperature and an increase in flow of blood in that extremity.

The rise in surface temperature of the extremities of subjects with thrombo-angiitis obliterans varied considerably among different persons

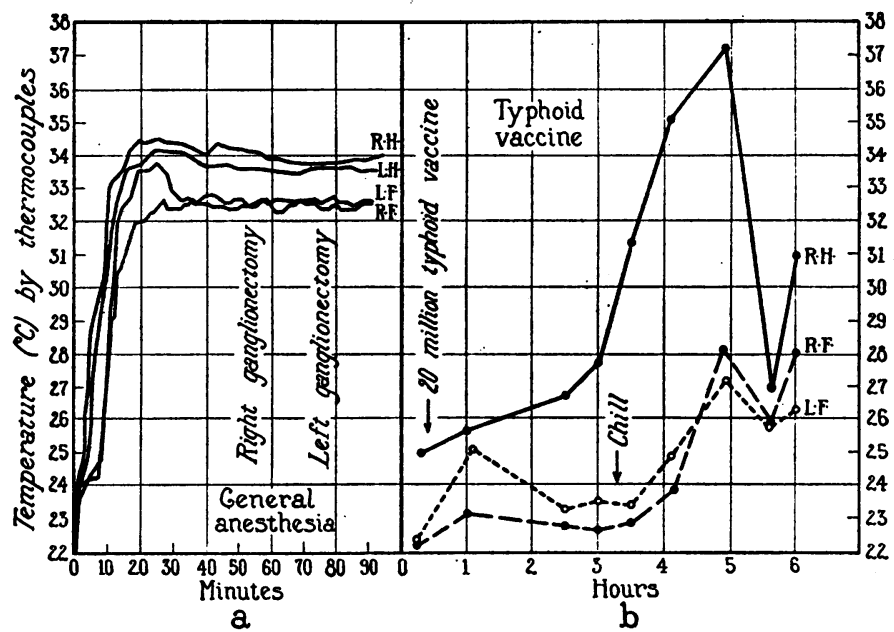

Fig. 2. Increase in Surface Temperature of the Hands and Feet of a Woman Aged Twenty-three Years Who Had Raynaud's Disease

Pulsations in the peripheral vessels were normal. $a$. The rise with general anesthesia. $b$. The rise obtained with intravenous injection of typhoid vaccine. Rise in mouth temperature was $1.1^{\circ} \mathrm{C}$. R. H., right hand; R. F., right foot; L. F., left foot, and L. H., left hand.

and corresponded clinically to the degree of occlusive vascular disease present (Figs. $3 a, 4 a, 5 a$ and $6 a$ ). In thrombo-angiitis obliterans, one is dealing primarily with an occlusive vascular disease which involves the extremities. Vasospastic disturbance in the collateral circulation is invariably present to some degree. Interruption of the sympathetic nerve supply to the affected extremities permits the maximal flow of blood through the collateral circulation, but it is obvious that the occluded vessels are not affected by this surgical procedure. In cases in which there is a small degree of occlusion and a large degree of superimposed vasospasm in the collateral circulation, the rise in surface temperature of the extremities parallels that observed in cases of Raynaud's disease. In moderately advanced cases of thrombo-angiitis obliterans, uniform rises in surface temperature of all four extremities were not observed, but in early cases the rise in surface temperature of the extremities may closely simulate that observed in Raynaud's disease, as illustrated in Figure $3 a$.

It is interesting to compare the various rises in temperature obtained with intravenous administration of typhoid vaccine in a given case and 


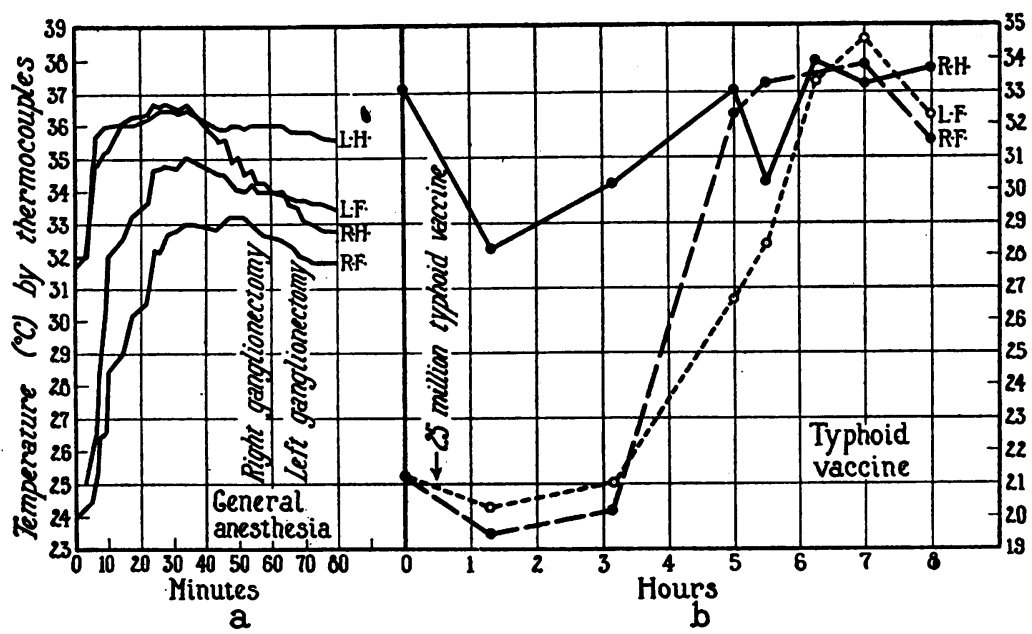

Fig. 3. Variation in Surface Temperatures of the Hands and Feet of a Man Aged Thirty-two Years Who Had Thrombo-angittis Obliterans

Pulsations in the right and left ulnar, radial, femoral and popliteal arteries were normal. In other arteries, pulsations were reduced as follows: in the right dorsalis pedis, 80 per cent; in the left dorsalis pedis, 65 per cent; in the right posterior tibial, 35 per cent, and in the left posterior tibial, 25 per cent. $a$. The rise with general anesthesia. $b$. The rise obtained with intravenous injection of typhoid vaccine. Rise in mouth temperature was $0.5^{\circ} \mathrm{C}$. R. H., right hand; R. F., right foot; L. F., left foot, and L. H., left hand.

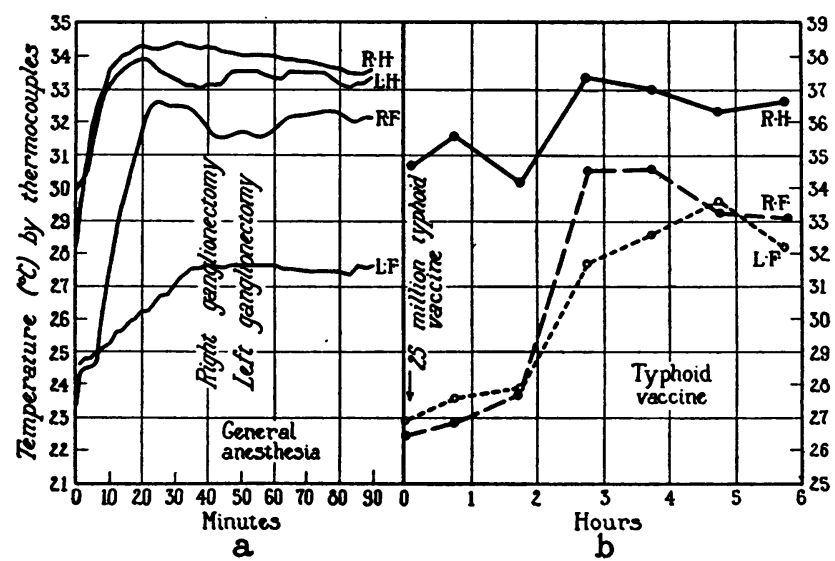

Fig. 4. Increase in Surface Temperature of the Hands and Feet of a Man Aged Forty Years Who Had Thrombo-angirtis Obliterans

The right and left ulnar, radial and femoral arteries pulsated normally. Pulsations in both popliteal arteries were reduced approximately 50 per cent; in the right and left dorsalis pedis and posterior tibial arteries pulsations were absent. Circulatory insufficiency was more marked in the left than in the right foot. $a$. The rise with general anesthesia. $b$. The rise obtained with intravenous injection of typhoid vaccine. Rise in mouth temperature was $1.3^{\circ}$ C. R. H., right hand; R. F., right foot; L. F., left foot, and L. H., left hand. 
that obtained following induction of general anesthesia. We have previously been of the opinion that similar temperature curves could be obtained. This, however, proved not to be true, for there was marked dissimilarity between the curves obtained following intravenous administration of typhoid vaccine and that following induction of general anesthesia (Figs. $2 b, 3 b, 4 b, 5 b$ and $6 b$ ). This in part may be due to the fact that some subjects are either refractory to or react poorly to intravenous administration of typhoid vaccine. The maximal flow of blood to the extremities occurred following both procedures, but it occurred more quickly after general anesthesia than after intravenous administration of typhoid vaccine. With general anesthesia, fewer fluctuations were observed after the surface temperature had reached its maximum. It

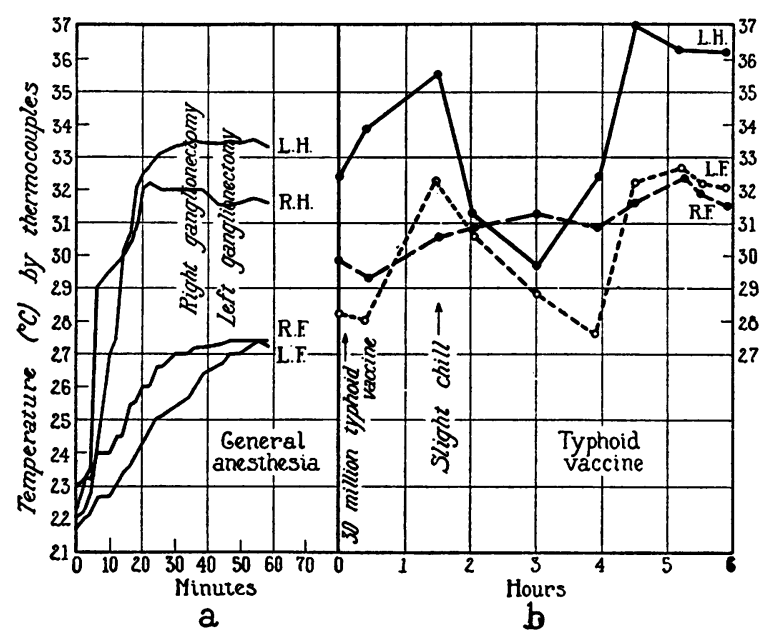

Fig. 5. Variation in Surface Temperature of the Hands and Feet of a Man Aged Twenty-nine Years Who Had Thrombo-angitis Obliterans

Pulsations in the right and left ulnar, radial, and femoral arteries, and in the right popliteal arteries, were normal; those in the left popliteal artery were reduced 25 per cent. Pulsations in the right and left dorsalis pedis arteries were reduced 40 per cent, and the right and left posterior tibial arteries were occluded. The circulatory insufficiency was the same in both feet. $a$. The rise with general anesthesia. $b$. The rise obtained with intravenous injection of typhoid vaccine. Rise in mouth temperature was $2.2^{\circ} \mathrm{C}$. R. H., right hand; R. F., right foot; L. F., left foot, and L. H., left hand.

was also evident that the maximal flow of blood to the extremities following general anesthesia occurred within twenty or thirty minutes and was not increased after that time. The fluctuation in the curves of surface temperature following intravenous injection of typhoid vaccine is accounted for, in part at least, by the chill which so frequently develops. Brown originally used typhoid vaccine intravenously to determine what he designated "the vasomotor index." $\mathrm{He}$ and Adson $(2,3)$ used this 
method for selecting subjects with Raynaud's disease and thromboangiitis obliterans for sympathetic ganglionectomy. It has proved to be a satisfactory means of selecting cases for this surgical procedure. In addition, the intravenous administration of typhoid vaccine or "fever therapy" undoubtedly represents the most important medical method of treatment for these diseases. This is particularly true with reference to thrombo-angiitis obliterans.

Other methods for selecting cases for sympathetic ganglionectomy are spinal anesthesia, which is applicable only to the lower extremities, and paravertebral injection of procaine in the cervicothoracic region for the upper extremities. Oral administration of alcohol, intramuscular injec-

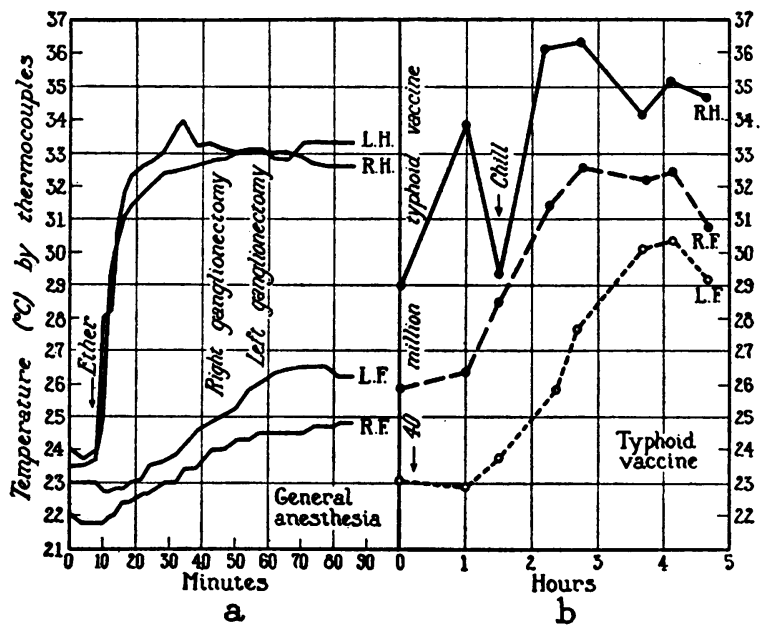

Fig. 6. Variation in Surface Temperature of Hands and Feet of a Man

Aged Thirty-two Years Who Had Thrombo-angirtis Obliterans

Pulsations in the right ulnar, radial, and femoral arteries, and in the left radial and femoral arteries, were normal. Pulsations were absent in the other palpable vessels. Circulatory insufficiency was more marked in the right than in the left foot. $a$. The rise with general anesthesia. $b$. The rise obtained with intravenous injection of typhoid vaccine. Rise in mouth temperature was $1^{\circ}$ C. R. H., right hand; R. F., right foot; L. F., left foot, and L. H., left hand.

tion of acetylcholine, application of heat, and many other procedures also have been used with varying degrees of success. The procedure of observing surface temperatures of the subject under anesthesia affords a satisfactory method for investigating the amount and extent of vasospastic disturbances particularly in the extremities of subjects with Raynaud's disease and thrombo-angiitis obliterans. If satisfactory rises in the surface temperature of the involved extremities are not observed after induction of anesthesia, it is evident that an additional rise will not develop after interruption of the sympathetic innervation. The same 
observations were made in investigating cases of vasospastic scleroderma and chronic infectious arthritis. Thermal changes under general anesthesia give valuable information from the prognostic standpoint in subjects with thrombo-angiitis obliterans. Accurate information can be obtained regarding the extent of the occlusive process in the extremities and the amount of superimposed vasospasm in the collateral circulation. With the history of the case, the physical examination, and the information derived from such a study, the prognosis in a given case can be estimated fairly adequately.

\section{SUMMARY AND CONCLUSIONS}

Continuous observations of surface temperature, before and during sympathetic ganglionectomy, were made on all four extremities of subjects with Raynaud's disease and thrombo-angiitis obliterans. An electromotive thermometer was used. The difference between these two diseases is well illustrated by this study. General anesthesia alone produced maximal vasodilation in Raynaud's disease and thrombo-angiitis obliterans. Severance of the sympathetic nerves did not cause additional vasodilation. In Raynaud's disease a prompt and uniform vasodilating response was observed in the peripheral vessels of all four extremities, but in thrombo-angiitis obliterans uniform response was absent because the occlusive process in the vessels of all four extremities is never the same in thrombo-angiitis obliterans.

The graphic records made with anesthesia at the time of operation serve as a check on preoperative studies, carried out with other vasodilating agents. If satisfactory rises in the surface temperature of the involved extremities are not observed after induction of general anesthesia, it is evident that an additional rise will not develop after interruption of the sympathetic innervation.

General anesthesia constitutes a satisfactory method for investigating thermal changes in cases of thrombo-angiitis obliterans, Raynaud's disease, vasospastic scleroderma, and arthritis, prior to and during sympathetic ganglionectomy. Valuable information may be obtained regarding the amount and extent of vasospasm in the vessels of the extremities, particularly in the collateral circulation of subjects with thromboangiitis obliterans, and the method gives accurate information regarding the degree of occlusive process in this group of cases. Vessels of patients who are refractory to, or react poorly to, other vasodilating agents will usually undergo maximal vasodilation when general anesthesia is administered.

\section{BIBLIOGRAPHY}

1. Adson, A. W., Personal communication to the authors.

2. Brown, G. E. and Adson, A. W., Am. J. M. Sc., 1925, clxx, 232. Calorimetric Studies of the Extremities Following Lumbar Sympathetic Ramisection and Ganglionectomy. 
3. Brown, G. E. and Adson, A. W., Arch. Neurol. and Psychiat., 1929, xxii, 322. Physiologic Effects of Thoracic and of Lumbar Sympathetic Ganglionectomy or Section of the Trunk.

4. Herrick, Julia F. and Baldes, E. J., Physics, 1931, i, 407. The ThermoStromuhr Method of Measuring Blood Flow.

5. Herrick, J. F., Essex, H. E. and Baldes, E. J., Am. J. Physiol., 1932, ci, 213. The Effect of Lumbar Sympathectomy on the Flow of Blood in the Femoral Artery of the Dog.

6. Morton, J. J. and Scott, W. J. M., J. Clin. Invest., 1930, ix, 235. The Measurement of Sympathetic Vasoconstrictor Activity in the Lower Extremities.

7. Royle, N. D., M. J. Australia, 1924, i, 77. A New Operative Procedure in the Treatment of Spastic Paralysis and its Experimental Basis.

8. Sheard, Charles, Am. J. Clin. Path., 1931, i, 209. The Electromotive Thermometer. An Instrument and a Method for Measuring Intramural, Intravenous, Superficial and Cavity Temperatures.

9. Sheard, Charles, Rynearson, E. H. and Craig, W. McK., J. Clin. Invest., 1932, xi, 183. Effects of Environmental Temperature, Anesthesia and Lumbar Sympathetic Ganglionectomy on the Temperatures of the Extremities of Animals. 